\title{
Transferability of Dipole Moment Derivatives: Calculation of Absolute Rotational Contributions for Symmetrical Molecules
}

\author{
J. H. G. Bode, W. M. A. Smit, ${ }^{1}$ and A. J. van Straten ${ }^{2}$ \\ Analytical Chemistry Laboratory, University of Utrecht, Croesestraat 77A, \\ 3522 AD Utrecht, The Netherlands
}

\begin{abstract}
It is shown that Polo's method for the calculation of the $\mathbf{D}^{-1}$ and $\mathbf{G}^{-1}$ matrices can be extended to the calculation of the absolute rotational contributions involved in the molecular dipole moment derivatives in terms of symmetry coordinates. The method applies to both stretching and bending modes. The method is outlined for $A B_{3}\left(C_{3 r}\right)$ molecules.
\end{abstract}

\section{INTRODUCTION}

In order to study the transferability of $\partial \mu / \partial S$ and other polar quantities from one molecule to another the absolute rotational contributions involved in the molecular dipole moment derivatives have to be known $(I, 2)$. Special reference molecules have been proposed to calculate such contributions for a number of symmetrical molecules $(1-3)$. However, as will be seen below, the use of heavy-isotope (1) and zero-isotope (3) reference molecules leads to absolute rotational corrections only for the stretching modes. Further, it has been shown that the absolute rotational contributions can be calculated directly from the bond direction changes involved in stretching modes $(4,5)$. Unfortunately, however, the absolute contributions involved in the bending modes cannot be obtained in this way as long as one cannot distinguish between the pure vibrational bond direction changes and those originating from the Eckart conditions.

In 1956 Polo (6) published a method for obtaining analytical expressions for the $\mathbf{D}^{-1}$ and $\mathbf{G}^{-1}$ matrix elements. His method was based on the use of pure geometrical symmetry distortions to which appropriate amounts of translation and rotation were added to satisfy the Eckart conditions. Obviously, the added amounts of rotation are directly related to the rotational contributions involved in the dipole moment derivatives. We therefore have investigated the possibility of extending Polo's method to the calculation of absolute rotational contributions. In the following it will be shown that absolute rotational contributions can be obtained for small symmetrical molecules based on the definition of a unique set of pure geometrical symmetry distortions.

${ }^{1}$ Author to whom correspondence should be addressed.

${ }^{2}$ Present address: Koninklijke/Shell Laboratorium, (Shell Research B.V.) Badhuisweg 3, Amsterdam, The Netherlands. 
THEORETICAL CONSIDERATIONS

The complete set of $3 N$ coordinates describing the normal modes of an $N$ atomic molecule can be defined as

$$
\left|\begin{array}{c}
\mathbf{S} \\
\hdashline \boldsymbol{\rho}
\end{array}\right|=\left|\frac{\mathbf{B}_{\mathrm{S}}}{-\boldsymbol{\beta}}\right| \mathbf{X}
$$

The inverse transformation is given by

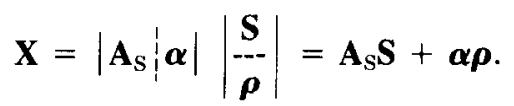

The $(3 N-6) S$ coordinates describe the internal symmetry distortions of the molecule, while the six $\rho$ coordinates describe the external modes, viz., the translations and rotations. In order to ensure orthogonality between the internal and external modes the Eckart conditions should be satisfied:

$$
\begin{aligned}
\boldsymbol{\beta} \mathbf{A}_{\mathrm{S}} & =\mathbf{0}, \\
\mathbf{B}_{\mathrm{S}} \boldsymbol{\alpha} & =\mathbf{0}
\end{aligned}
$$

In general, the internal symmetry coordinates $S$ defined by Eqs. (1)-(3) do not represent pure geometrical distortions but include small amounts of translation and rotation in order to satisfy the conditions of Eq. (3). A pure geometrical symmetry distortion may be defined by a particular set of Cartesian displacement vectors such as to cause a unit change in $S_{i}$ while all other internal symmetry coordinates remain unchanged. The components of the vectors of that particular set are the elements of the $i$ th column of the $\mathbf{A}_{\mathrm{Sg}}$ matrix, given by

$$
\mathbf{X}=\mathbf{A}_{\mathbf{S g}} \mathbf{S}_{\mathbf{g}} .
$$

However, such sets can be chosen in an infinite number of ways. If additional conditions leading to a unique choice of the pure geometrical symmetry distortions $S_{\mathrm{g}}$ exist, the absolute amounts of translation and rotation involved in the $S$ coordinates can be easily obtained. For the moment let us suppose that such conditions can be found. In matrix notation the Polo definition of $\mathbf{A}_{\mathrm{Sg}}$ reads

$$
\mathbf{A}_{S}=\mathbf{A}_{\mathrm{Sg}}-\boldsymbol{\alpha} \boldsymbol{\beta} \mathbf{A}_{\mathrm{Sg}},
$$

which corresponds to Eq. (II, 8) of Polo's paper (6). The product-matrix $\boldsymbol{\beta} \mathbf{A}_{\mathrm{Sg}}$ represents the amounts of translation and rotation set up in the molecule by the pure geometrical distortions, while $\boldsymbol{\alpha} \boldsymbol{\beta} \mathbf{A}_{\mathrm{Sg}}$ gives the Cartesian displacements corresponding to these translations and rotations.

From the condition

$$
\mathbf{B}_{\mathrm{S}} \mathbf{A}_{\mathrm{S}}=\mathbf{E}_{3 N-6}
$$

one easily arrives, viewing Eqs. (3) and (5), at

$$
\mathbf{B}_{\mathrm{S}} \mathbf{A}_{\mathrm{Sg}}=\mathbf{E}_{3 N-6} \text {, }
$$

in accordance with the definition of $\mathbf{A}_{\mathrm{Sg}}$. Multiplying Eq. (5) from the left by 
the $\mathbf{P}_{\mathbf{X}}$ polar tensor (7) gives

$$
\mathbf{P}_{\mathrm{X}} \mathbf{A}_{\mathrm{Sg}}=\mathbf{P}_{\mathrm{X}} \mathbf{A}_{\mathrm{S}}+\mathbf{P}_{\mathrm{X}} \boldsymbol{\alpha} \boldsymbol{\beta} \mathbf{A}_{\mathrm{Sg}}
$$

Equation (8) can be rewritten as

$$
\mathbf{P}_{\mathrm{Sg}}=\mathbf{P}_{\mathrm{S}}+\mathbf{P}_{\rho} \boldsymbol{\beta} \mathbf{A}_{\mathrm{Sg}} \text {. }
$$

The elements of the polar tensor $\mathbf{P}_{\mathrm{Sg}}$ are the desired dipole moment derivatives in terms of pure geometrically defined symmetry coordinates. It is hoped that these quantities are transferable from one molecule to another when corresponding modes of the same groups in both molecules are considered.

In accardance with common usage, let us define the product-matrix

$$
\mathbf{V}=-\mathbf{P}_{\rho} \boldsymbol{\beta} \mathbf{A}_{\mathrm{Sg}},
$$

which contains the absolute rotational contributions that should be subtracted from the observed dipole moment derivatives collected in $\mathbf{P}_{\mathrm{S}}$ in order to obtain the transferable derivatives of $\mathbf{P}_{\mathrm{Sg}}$. Thus the elements of $\mathbf{V}$ may be referred to as the absolute rotational corrections. Equation (10) can be evaluated to obtain expressions for the individual rotational corrections. Since translational motions do not change the molecular dipole moment $\mu, \mathbf{P}_{\rho}$ can be taken as (5)

$$
\mathbf{P}_{\rho}=\mathbf{P}_{\mathrm{X}} \boldsymbol{\alpha}=\left[\begin{array}{cccccc}
0 & 0 & 0 & 0 & \mu_{0 z} & -\mu_{0 y} \\
0 & 0 & 0 & -\mu_{0 z} & 0 & \mu_{0 x} \\
0 & 0 & 0 & \mu_{0 y} & -\mu_{0 x} & 0
\end{array}\right],
$$

the $\alpha$ matrix being normalized by $(1,5)$

$$
\tilde{\boldsymbol{\alpha}} \mathbf{M} \boldsymbol{\alpha}=\mathbf{N}^{-1} .
$$

Note that the form of $\mathbf{P}_{\rho}$ given by Person and Newton (7) is slightly different due to the different normalization they use for the $\boldsymbol{\alpha}$ matrix, viz., $\tilde{\boldsymbol{\alpha}}^{\prime} \mathbf{M} \boldsymbol{\alpha}^{\prime}=\mathbf{E}$, leading to the following relationship between their $\boldsymbol{\alpha}^{\prime}$ matrix and our $\boldsymbol{\alpha}$ matrix: $\boldsymbol{\alpha}^{\prime}=\boldsymbol{\alpha} \mathbf{N}^{1 / 2}$, the $\mathbf{N}$ matrix being a $6 \times 6$ diagonal matrix with the elements $M^{-1}$, $M^{-1}, M^{-1}, I_{x}^{-1}, I_{y}^{-1}, I_{z}^{-1}$, where $M$ denotes the molecular mass and $I_{x}, I_{y}$, and $I_{z}$ are the principal moments of inertia $(1,5)$. The rotational correction, however, is not affected by the normalization chosen since $\mathbf{V}=-\mathbf{P}_{X} \boldsymbol{\alpha}^{\prime} \boldsymbol{\beta}^{\prime} \mathbf{A}_{\mathrm{Sg}}=-\mathbf{P}_{X} \boldsymbol{\alpha} \mathbf{N}^{1 / 2}$ $\times \mathbf{N}^{-1 / 2} \boldsymbol{\beta} \mathbf{A}_{\mathrm{Sg}}=-\mathbf{P}_{X} \boldsymbol{\alpha} \boldsymbol{\beta} \mathbf{A}_{\mathrm{Sg}}=-\mathbf{P}_{\rho} \boldsymbol{\beta} \mathbf{A}_{\mathrm{Sg}}$.

Viewing Eq. (11), one easily arrives at

$$
\begin{aligned}
& V_{x i}=-\mu_{0 z}\left(\beta A_{\mathrm{Sg}}\right)_{y i}+\mu_{0 y}\left(\beta A_{\mathrm{Sg}}\right)_{z i}, \\
& V_{y i}=\mu_{0 z}\left(\beta A_{\mathrm{Sg}}\right)_{x i}-\mu_{0 x}\left(\beta A_{\mathrm{Sg}}\right)_{z i}, \\
& V_{z i}=-\mu_{0 y}\left(\beta A_{\mathrm{Sg}}\right)_{x i}+\mu_{0 x}\left(\beta A_{\mathrm{Sg}}\right)_{y i},
\end{aligned}
$$

where $\left(\beta A_{\mathrm{Sg}}\right)_{\xi i}(\xi=x, y, z)$ denotes the product-matrix element originating from multiplication of the $\beta$-matrix row corresponding to the rotational coordinate $\rho_{\xi}$ and the $i$ th column of $\mathbf{A}_{\mathrm{Sg}}$, while $\mu_{0 \xi}$ denotes the $\xi$-component of the molecular equilibrium dipole moment $\mu_{0} \cdot V_{\xi i}$ is the rotational correction belonging to $\partial \mu_{\xi} / \partial S_{i}$.

Equation (13) can be further evaluated by making use of the general form of 
the $\beta$ matrix ( 1 ) normalized by

$$
\boldsymbol{\beta} \mathbf{M}^{-1} \tilde{\boldsymbol{\beta}}=\mathbf{N}
$$

and the method given by Polo (6) to determine the $\mathbf{A}_{\mathrm{Sg}}$ matrix elements. In the next section expressions for the absolute rotational corrections will be derived for $A B_{3}\left(C_{3 v}\right)$ molecules.

\section{APPLICATION TO $A B_{3}\left(C_{3 n}\right)$ MOLECULES}

The Cartesian axes, the internal coordinates, and the bond unit vectors for the pyramidal $A B_{3}$ molecule are given in Fig. 1. The nonzero $\rho$-vectors in terms of the internal coordinates are (6)

$$
\begin{array}{ll}
R_{1}=\Delta r_{41,} & \boldsymbol{\rho}_{11}=k_{11} \mathbf{e}_{41}, \\
& \rho_{12}=\rho_{13}=\rho_{14}=-k_{14} \mathbf{e}_{41},
\end{array}
$$

and similar expressions for $R_{2}=\Delta r_{42}$ and $R_{3}=\Delta r_{43}$;

$$
\begin{aligned}
R_{4}=\Delta \alpha_{1}, \quad \boldsymbol{\rho}_{42} & =\frac{k_{42}\left(\mathbf{r}_{42} \times \mathbf{e}_{41}\right) \sin \alpha}{\mathbf{e}_{41}\left(\mathbf{e}_{42} \times \mathbf{e}_{43}\right)}, \\
\boldsymbol{\rho}_{43} & =\frac{k_{43}\left(\mathbf{r}_{43} \times \mathbf{e}_{41}\right) \sin \alpha}{\mathbf{e}_{41}\left(\mathbf{e}_{42} \times \mathbf{e}_{43}\right)},
\end{aligned}
$$

and similar expressions for $R_{5}=\Delta \alpha_{2}$ and $R_{6}=\Delta \alpha_{3}$. The symmetry coordinates are

$$
\begin{aligned}
A_{1}: \quad S_{1} & =3^{-1 / 2}\left(R_{1}+R_{2}+R_{3}\right), \\
S_{2} & =3^{-1 / 2}\left(R_{4}+R_{5}+R_{6}\right) \\
E: \quad S_{3 x} & =6^{-1 / 2}\left(2 R_{1}-R_{2}-R_{3}\right), \\
S_{4 x} & =6^{-1 / 2}\left(2 R_{4}-R_{5}-R_{6}\right) .
\end{aligned}
$$

The constants $k$ in the expressions for the $\rho$-vectors can be chosen arbitrarily

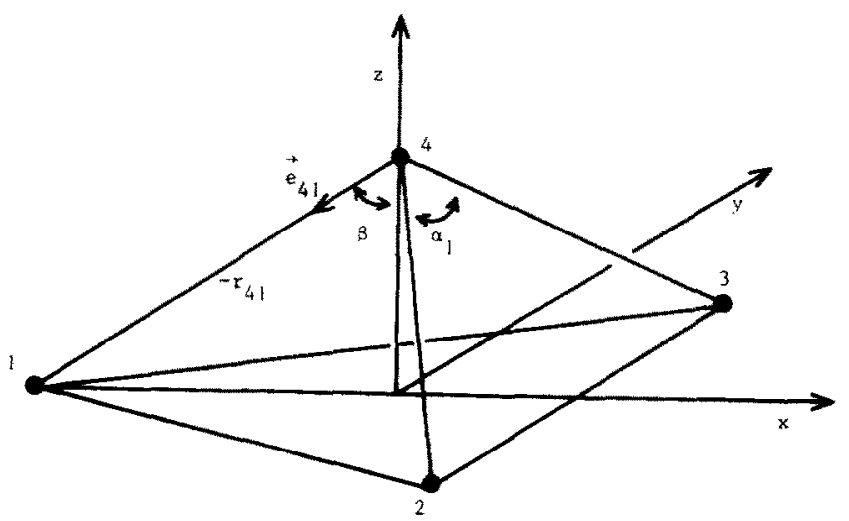

Fic. 1. The $A B_{3}$ molecule. Definition of internal coordinates. Cartesian axes, and bond unit vectors. 
within certain conditions. For the stretching mode $R_{1}$ these conditions are:

$$
\begin{aligned}
& k_{11}+k_{14}=1, \\
& k_{12}=k_{13}=k_{14} .
\end{aligned}
$$

Similar conditions hold for $R_{2}$ and $R_{3}$. Moreover, in order to ensure a zero rotational correction for the $S_{1}$ mode, the $k$-values have to satisfy the relations

$$
k_{11}=k_{22}=k_{33} \text {. }
$$

The values actually chosen are $k_{11}=k_{22}=k_{33}=1$, and thus

$$
k_{14}=k_{24}=k_{34}=0 \text {. }
$$

The same choice has been made for the $S_{3 x}$ mode. The only choice leading to a zero rotational contribution involved in the $S_{2}$ bending mode is:

$$
k_{42}=k_{43}=k_{51}=k_{53}=k_{61}=k_{62}=1 / 2 .
$$

The same choice has to be made for the $S_{4 x}$ mode.

This leads to the following $\rho$-vector components for $S_{3 x}$ :

$$
\begin{aligned}
& S_{3 x}: \rho_{31}=\left(-1 / 3\left(6^{1 / 2}\right) \sin \beta, 0,-1 / 3\left(6^{1 / 2}\right) \cos \beta\right), \\
& \rho_{32}=\left(-1 / 12\left(6^{1 / 2}\right) \sin \beta, 1 / 4\left(2^{1 / 2}\right) \sin \beta, 1 / 6\left(6^{1 / 2}\right) \cos \beta\right), \\
& \rho_{33}=\left(-1 / 12\left(6^{1 / 2}\right) \sin \beta,-1 / 4\left(2^{1 / 2}\right) \sin \beta, 1 / 6\left(6^{1 / 2}\right) \cos \beta\right), \\
& \rho_{34}=(0,0,0) .
\end{aligned}
$$

The components of the position vectors $\mathbf{r}_{0 \alpha}$ in the principal-axis system can be derived to be

$$
\begin{aligned}
& \mathbf{r}_{01}=\left(-r \sin \beta, 0, \frac{-m_{A} r \cos \beta}{M}\right), \\
& \mathbf{r}_{02}=\left(1 / 2 r \sin \beta,-1 / 2\left(3^{1 / 2}\right) r \sin \beta, \frac{-m_{A} r \cos \beta}{M}\right), \\
& \mathbf{r}_{03}=\left(1 / 2 r \sin \beta, 1 / 2\left(3^{1 / 2}\right) r \sin \beta, \frac{-m_{A} r \cos \beta}{M}\right), \\
& \mathbf{r}_{04}=\left(0,0, \frac{3 m_{B} r \cos \beta}{M}\right) .
\end{aligned}
$$

This leads to the following expression for the $\left(\beta A_{\mathrm{Sg}}\right)_{y, 3 x}$ element:

$$
\left(\beta A_{\mathrm{Sg}}\right)_{y, 3 x}=\frac{-3 / 2\left(6^{1 / 2}\right) m_{B}^{2} r \sin \beta \cos \beta}{M I_{y}},
$$

and thus the rotational correction for the $S_{3 x}$ mode can be written as

$$
V_{x, 3 x}=\frac{3 / 2\left(6^{1 / 2}\right) m_{B}^{2} r \sin \beta \cos \beta \mu_{0}}{M I_{y}},
$$


in full accordance with the expression obtained from the bond direction changes $(4,5)$.

The $\rho$-vectors for $S_{4 x}$ are found to be

$$
\begin{aligned}
S_{4 x}: \quad \boldsymbol{\rho}_{41} & =\left(\frac{r \sin \alpha}{3\left(6^{1 / 2}\right) \sin \beta}, 0, \frac{-r \sin \alpha}{3\left(6^{1 / 2}\right) \cos \beta}\right), \\
\boldsymbol{\rho}_{42} & =\left(\frac{-2 r \sin \alpha}{3\left(6^{1 / 2}\right) \sin \beta}, \frac{-r \sin \alpha}{3\left(2^{1 / 2}\right) \sin \beta}, \frac{r \sin \alpha}{6\left(6^{1 / 2}\right) \cos \beta}\right), \\
\boldsymbol{\rho}_{43} & =\left(\frac{-2 r \sin \alpha}{3\left(6^{1 / 2}\right) \sin \beta}, \frac{r \sin \alpha}{3\left(2^{1 / 2}\right) \sin \beta}, \frac{r \sin \alpha}{6\left(6^{1 / 2}\right) \cos \beta}\right), \\
\boldsymbol{\rho}_{44} & =(0,0,0) .
\end{aligned}
$$

The resulting expression for the absolute rotational contribution for $S_{4, x}$ reads

$$
V_{x, 4 x}=\frac{m_{B} r^{2} \sin \alpha\left(3 \sin ^{2} \beta m_{B}-2 \cos \alpha m_{A}\right) \mu_{0}}{2\left(6^{1 / 2}\right) \sin \beta \cos \beta M I_{y}}
$$

\section{DISCUSSION AND CONCLUSIONS}

The expressions for the absolute rotational contributions obtained from the definition of pure geometrical distortions lead, for the asymmetrical stretching modes of pyramidal $A B_{3}$ molecules, to values identical to those obtained both from the bond direction changes $(4,5)$ and from the use of heavy-isotope reference molecules $(l)$. However, the value obtained from Eq. (24) for the asymmetrical $A B_{3}$ bending mode differs substantially from the one obtained from the heavy-isotope method. Thus, the heavy-isotope molecule does not behave rotation-free with regard to the $S_{4, x}$ mode, which leads to the general conclusion that the zero- and heavy-isotope reference molecules are not rotationfree with regard to bending modes. Hence rotational corrections for bending modes obtained from the use of these reference molecules should not be referred to as absolute corrections. This means that reference molecules can only be used for the calculation of relative rotational corrections. Such corrections may be used to compare dipole moment derivatives within an isotopic series $(8,9)$. However, in such cases the parent molecule (the molecule containing the most abundant isotopes) seems to be the obvious reference $(9)$.

The construction of the $\mathbf{A}_{\mathrm{Sg}}$ matrix elements from the components of Polo's pure geometrical $\rho$-vectors leads to a very useful method for the calculation of absolute rotational contributions without making use of a reference molecule. In the Appendix of his paper Polo has given the expressions for the $\boldsymbol{\rho}$-vectors for all kinds of internal coordinates. For the simple symmetrical $A B_{3}$ molecule the arguments to fix the $k$-values of the $\rho$-vectors to particular values are straightforward. If proper conditions for the $k$-values can be found for all kinds of coordinates in any molecule the absolute rotational contributions can be easily obtained.

In a forthcoming paper the generalization of this method will be discussed and applied to a number of asymmetrical molecules. 


\section{ACKNOWLEDGMENT}

Helpful discussions with Dr. F. G. Dijkman are gratefully acknowledged.

ReCEIVED: May 26, 1978

\section{REFERENCES}

1. A. J. van Straten and W. M. A. Smit, J. Mol. Spectrose. 56, 484-493 (1975).

2. M. Gussoni and S. Abbate, J. Mol. Spectrosc. 62, 53-59 (1976); M. Gussoni and S. AbBate, J. Chem. Phys. 65, 3439-3445 (1976).

3. A. D. Dickson, I, M. Mills, ANd B. L. CRAWford, JR., J. Chem. Phys. 27, 445-455 (1957).

4. A. J. van Straten And W. M. A. Smit, J. Mol. Spectrosc. 62, 297-312 (1976).

5. A. J. Van Straten and W. M. A. Smit, J. Mol. Spectrosc. 65, 202-218 (1977).

6. S. R. Polo, J. Chem. Phys. 24, 1133-1138 (1956).

7. W. B. Person and J. H. Newton, J. Chem. Phys. 61, 1040-1049 (1974).

8. B. L. Crawford, JR., J. Chem. Phys. 20, 977-981 (1952).

9. W. M. A. Smit, J. H. G. Bode, and A. J. van Straten, J. Mol. Spectrosc, 72, 180-181 (1978). 